\title{
Sífilis Materna e Congênita em Rondônia: casos notificados de 2010 a 2015
}

\author{
Maternal and congenital syphilis in Rondônia: cases reported of 2010 to 2015 \\ Sífilis materna y congénita en Rondônia: casos reportados de 2010 a 2015
}

Jeanne Lúcia Gadelha Freitas ${ }^{1 *}$, Priscilla Perez da Silva Pereira ${ }^{1}$, Kátia Fernanda Alves Moreira ${ }^{1}$,

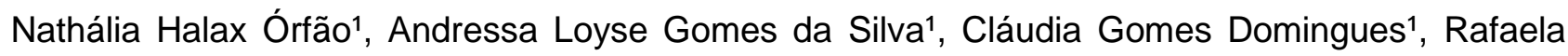
Caroline Brito Garcia1.

\section{RESUMO}

Objetivo: Analisar casos de sífilis congênita notificados e estimar as taxas de incidência e mortalidade da doença em Rondônia de 2010 a 2015. Métodos: Estudo descritivo com dados coletados das notificações de agravo compulsório do Sistema de Informação sobre Nascidos Vivos e do Sistema de Informação sobre Mortalidade. Foram analisadas as frequências por biênios, cálculo de incidência e taxa de mortalidade. Resultados: Dos 345 casos de sífilis congênita, a incidência variou de 0,7 a 3,7 casos/1,000 nascidos vivos e a taxa de mortalidade de 0,0 a 10,7/100,000 nascidos vivos. Houve predomínio de gestantes adultas, pardas, com ensino fundamental, residentes na zona urbana. A maioria realizou pré-natal, mas teve diagnóstico pelo VDRL reagente no parto/curetagem e teste confirmatório. Metade das gestantes tiveram tratamento inadequado e $58,6 \%$ dos parceiros não foram tratados. Nas crianças, $78,8 \%$ tiveram VDRL reagente, maioria sem exame do liquor $(76,2 \%)$, assintomáticas $(76 \%)$ e com desfecho de sífilis congênita recente (82,6\%). Conclusão: $O$ diagnóstico da sífilis congênita apenas no parto/puerpério sugere fragilidade do pré-natal, o que compromete o controle do agravo. Os atuais resultados poderão auxiliar gestores e profissionais de saúde no manejo dessa doença.

Palavras-chave: Sífilis congênita, Gestantes, Saúde Materno-Infantil.

\begin{abstract}
Objectives: To analyze reported cases of silence and to estimate as disease incidence and mortality rates in Rondônia from 2010 to 2015. Methods: A descriptive study with data collected from reports of compulsory aggravation of the Information System on Live Births and the Mortality Information System. They were analyzed at frequencies by biênios, calculation of incidence and rate of mortality. Results: Of the 345 cases of congenital syphilis, an incidence ranged from 0.7 to 3.7 cases / 1000 live births and the mortality rate from 0.0 to $10.7 / 100,000$ live births. There was a predominance of adult pregnant women, with a primary education, living in the urban area. Most performed prenatal care but were diagnosed by the VDRL reagent at delivery / curettage and confirmatory testing. Half of the pregnant women had poor treatment and $58.6 \%$ of the partners were not treated. In the children, $78.8 \%$ had VDRL reagent, more without liqueur exam (76.2\%), asymptomatic (76\%) and with outcome of recent congenital syphilis (82.6\%). Conclusion: The diagnosis of congenital syphilis only does not give birth / puerperium suggests prenatal fragility, which is the problem of the control of the disease. The current resulting help managers and health professionals in the management of the disease.
\end{abstract}

Key words: Congenital Syphilis, Pregnant Women, Maternal and Child Health.

${ }^{1}$ Universidade Federal de Rondônia (UNIR), Porto Velho-Rondônia. *E-mail: jeannegadelha@unir.br 


\section{RESUMEN}

Objetivos: Analizar casos de sífilis congénita notificados y estimar las tasas de incidencia y mortalidad de la enfermedad en Rondônia de 2010 a 2015. Métodos: Estudio descriptivo con datos recolectados de las notificaciones de agravamiento obligatorio del Sistema de Información sobre Nacidos Vivos y del Sistema de Información sobre mortalidad. Se analizaron las frecuencias por bienios, cálculo de incidencia y tasa de mortalidad. Resultados: De los 345 casos de sífilis congénita, la incidencia varió de 0,7 a 3,7 casos / 1.000 nacidos vivos y la tasa de mortalidad de 0,0 a 10,7 / 100,000 nacidos vivos. Se observó predominio de gestantes adultas, pardas, con enseñanza fundamental, residentes en la zona urbana. La mayoría realizaron prenatal, pero tuvo diagnóstico por el VDRL reactivo en el parto / curetaje y prueba confirmatoria. La mitad de las gestantes tuvieron un tratamiento inadecuado y el $58,6 \%$ de los socios no fueron tratados. En los niños, $78,8 \%$ tuvieron VDRL reactivo, mayoría sin examen del licor (76,2\%), asintomáticos (76\%) y con desenlace de sífilis congénita reciente $(82,6 \%)$. Conclusión: El diagnóstico de la sífilis congénita sólo en el parto / puerperio sugiere fragilidad del prenatal, lo que compromete el control del agravio. Los resultados actuales pueden ayudar a gestores y profesionales de la salud en el manejo de esta enfermedad.

Palabras clave: Sífilis congénita; Sífilis gestacional; Salud Materno-Infantil.

\section{INTRODUÇÃO}

A sífilis é uma infecção sexualmente transmissível, causada pela bactéria Treponema pallidum. Sua ocorrência na gravidez pode resultar em sífilis congênita (SC) o que pode causar morte fetal precoce, baixo peso ao nascer, parto prematuro ou infecção no recém-nascido exposto (WHO, 2017; WANG et al., 2019). A SC ocorre quando a gestante com sífilis não é tratada ou tratada inadequadamente, transmitindo a infecção verticalmente ao concepto pela via transplacentária (GUERRERO M e LUCIANI K, 2019).

A despeito da eficácia do diagnóstico e tratamento para prevenir a SC em virtude dos baixos custos e efetividade, a transmissão de mãe para filho ainda é um problema de saúde pública em todo o mundo. Países como Canadá, Estados Unidos, Chile e Cuba obtiveram taxas próximas à eliminação da transmissão vertical (TV) da sífilis, porém nas Américas, desde a década de 2010, cerca de 164 a 344 mil crianças por ano nasceram com a infecção (WHO, 2017). A sífilis materna pode ser rastreada e facilmente evitada, mas a implementação de medidas práticas nos sistemas de saúde, ainda são difíceis de serem incorporadas, como por exemplo a adesão ao teste rápido duplo para HIV e Sífilis (SHAHROOK S, et al., 2014).

Em países subdesenvolvidos como o Brasil, a sífilis materna está associada à cor, ao baixo nível socioeconômico e de escolaridade, aos antecedentes de risco obstétrico, ao início tardio do pré-natal e ao número insuficiente de consultas (BRASIL, 2018). Por sua vez, a SC está associada ao manejo inadequado dos casos com perda de oportunidade de diagnóstico e tratamento, à ausência de aconselhamento e tratamento do parceiro e ao tratamento incorreto dos casos diagnosticados (SILVA LMC, et al., 2019).

A eliminação da SC é uma prioridade mundial estabelecida pela Organização Mundial de Saúde e a Organização Pan-Americana da Saúde porque apesar dos esforços governamentais, das organizações profissionais e da sociedade civil, a maioria das infecções maternas por sífilis permanecem não identificadas e não tratadas adequadamente (WHO, 2017). No Brasil, desde setembro de 2016, a meta pactuada junto a OPAS/OMS em 2010, foi estendida com um plano de ação para intensificar as medidas de prevenção e tratamento, visando eliminar a doença até 2030, priorizando as regiões Norte e Nordeste (BRASIL, 2018).

Isso porque no Brasil das últimas décadas o coeficiente de incidência de SC saltou de 2,4 casos/1.000 nascidos vivos (2010) para 8,6 casos/1.000 nascidos vivos (2017). Em 2016 a 2017 houve um aumento de $16,4 \%$ nas notificações, com destaque da elevada taxa de incidência na região Norte $(2,1$ casos para 4,4 casos/1.000 nascidos vivos). Nessa região, quase metade dos casos $(49,7 \%)$ foram identificados no 3 은 trimestre da gestação, expondo graves fragilidades da assistência materno-infantil, sobretudo pela baixa qualificação da atenção ao pré-natal, especialmente no manejo das Infecções Sexualmente Transmissíveis (IST) visando à detecção e tratamento precoce (BRASIL, 2018; BOTTURA BR, et al., 2019). 
Avaliações mais amplas sobre a assistência ao pré-natal, realizado em 252 municípios que compõem a Amazônia Legal e Nordeste do país, revelou níveis muito baixos de adequação ao pré-natal e ao parto. Rondônia como um dos oito estados que compõem esta região, apresentou naquele ano, um pífio desempenho no pré-natal $(4,1 \%)$ e parto $(0,2 \%)$ o que indica iniquidades regionais específicas à saúde materno infantil na Amazônia Legal e no Nordeste do Brasil (LEAL MC, et al., 2015).

Em Rondônia, estudos sobre perfil da SC na capital e interior do estado, indicaram elevado aumento dos casos e, embora relevantes, não expressam a real magnitude da doença (MAEDA ATN, et al., 2018, SOUZA AS e SAMPAIO LJ, 2018; MOREITA KFA, et al., 2017). Ampliar a investigação da incidência, mortalidade, tratamento e diagnóstico da sífilis no pré-natal na região é de fundamental importância para a gestão na esfera estadual e municipal, já que a doença é prevenível e com diagnóstico e tratamento eficazes.

Com base no exposto, o estudo tem como objetivos, analisar os casos de sífilis congênita notificados e estimar as taxas de incidência e de mortalidade pela doença, no estado de Rondônia, de 2010 a 2015.

\section{MÉTODOS}

Trata-se de um estudo descritivo com abordagem quantitativa de coleta retrospectiva, que utilizou como principal fonte de dados, todas as fichas de agravos de notificação compulsória de sífilis materna e sífilis congênita, disponíveis no Sistema de Informação de Agravos de Notificação (SINAN).

A coleta de dados das fichas individuais foi realizada no Núcleo Estadual de IST, Aids e Hepatites Virais da Agência Estadual de Vigilância em Saúde de Rondônia (AGEVISA-RO) situada no município de Porto Velho. Esta agência realiza o monitoramento de todas as fichas de agravos de notificação compulsória provenientes dos 52 municípios deste Estado.

O levantamento e seleção das fichas foram realizados após dupla revisão e correção das discrepâncias e/ou incompletudes das fichas pelos funcionários da AGEVISA visando garantir a qualidade dos dados. Também foram utilizadas as bases do Sistema de Informação Sobre Nascidos Vivos (SINASC) e Sistema de Informações sobre Mortalidade (SIM) para realização dos cálculos da incidência e taxa de mortalidade por SC. Foram considerados também os ajustes na base de dados no SINASC para o ano de 2011, quando o formulário da DNV sofreu modificações em algumas variáveis. Em Rondônia, foi utilizado os formulários novos ( $58 \%$ dos registros) e antigos (42\% dos registros) o que fez com que o estado atingisse uma razão de 90 e $100 \%$ na coleta das notificações sem prejuízos no cruzamento dos dados do recorte histórico analisado.

Foram incluídos todos os casos de sífilis em gestantes e crianças, notificados no estado no período de janeiro de 2010 a dezembro de 2015 e excluídas as fichas de notificações com preenchimento incompleto.

As variáveis estudadas foram:

1. Sociodemográficas - faixa etária materna (13-19 anos, 20-24 anos e 25- 44 anos); corda pele materna (parda, branca, preta e ignorada); escolaridade materna (ensino fundamental, ensino médio, ensino superior e ignorado); local de residência (urbana e rural).

2. Sobre o diagnóstico materno - realizou pré-natal (sim; não; ignorado); momento do diagnóstico de sífilis (pré-natal, parto/curetagem, após o parto, ignorado); VDRL da mãe no parto/curetagem (reagente, não reagente, não realizado, ignorado); Teste treponêmico confirmatório da mãe no parto/curetagem (reagente, não reagente, não realizado, ignorado).

3. Sobre o diagnóstico da criança - VDRL da criança (reagente, não reagente, não realizado, ignorado); Teste treponêmico de líquor da criança (reagente, não reagente, não realizado, ignorado); diagnóstico clínico da criança (assintomático, sintomático, não se aplica e ignorado); Diagnóstico final da criança (SC recente, descartados).

4. Sobre o tratamento da gestante (adequado, inadequado, não realizado, ignorado); tratamento do parceiro (adequado, inadequado, não realizado, ignorado); utilizou-se como critério de tratamento inadequado da gestante com sífilis: qualquer medicamento que não seja a penicilina benzatina; ou tratamento incompleto, 
mesmo tendo sido feito com penicilina benzatina; ou tratamento inadequado para a fase clínica da doença; ou instituição de tratamento dentro do prazo em até 30 dias antes do parto; ou parceiro(s) sexual (is) com sífilis não tratado(s) ou tratado(s) inadequadamente (BRASIL, 2019).

\section{Evolução dos casos - (vivos; óbitos por SC; óbitos por outras causas; ignorado)}

Os dados coletados foram tabulados no programa Microsoft Excel $2010 \AA$ e a análise descritiva realizada por meio de distribuição de frequência, cálculo da incidência (número de casos novos de SC em menores de um ano residentes em determinado espaço geográfico, dividido pelo número de nascidos vivos de mães residentes da mesma área no período considerado multiplicado por 1000) e taxa de mortalidade (razão entre o número de óbitos por SC para cada 100.000 nascidos vivos, no espaço geográfico em um determinado período).

O estudo faz parte do Projeto Matriz "Estudo Sobre Morbidades em Rondônia" do Centro de Estudos e Pesquisa em Saúde Coletiva (CEPESCO), aprovado pelo Comitê de Ética e Pesquisa (CEP) da Fundação Universidade Federal de Rondônia (UNIR) sob o número 1.205.923 para cumprimento da regulamentação de pesquisa com seres humanos, de acordo com a Resolução no 466/CNS/2012.

\section{RESULTADOS}

De 2010 a 2015, foram notificados 353 casos de sífilis congênita (SC) em Rondônia coletados dos 52 municípios notificadores. Destes, oito casos foram excluídos por não atender os critérios previamente estabelecidos, resultando em 345 casos notificados elegíveis para análise do estudo.

A Figura 1 mostra que as taxas de incidência de SC variaram de 0,7 casos/1.000 nascidos vivos em 2010 a 2,9 casos/1.000 nascidos vivos em 2015. O ano de 2014 teve a maior incidência (3,7 casos/ 1.000 nascidos vivos). A taxa de mortalidade por SC em 2010 e 2012 foi zero (0,0/100.000 nascidos vivos). Entretanto, as maiores taxas ocorreram em anos intercalados de $2011(3,6), 2013(3,6), 2014(7,2)$ e $2015(10,7)$ de óbitos para cada 100.000 nascidos vivos, um aumento de $7,1 \%$ na taxa de mortalidade no intervalo de apenas três anos (2013-2015) (Figura 1).

Figura 1 - Taxa de incidência e mortalidade por sífilis congênita, Rondônia, 2010-2015.

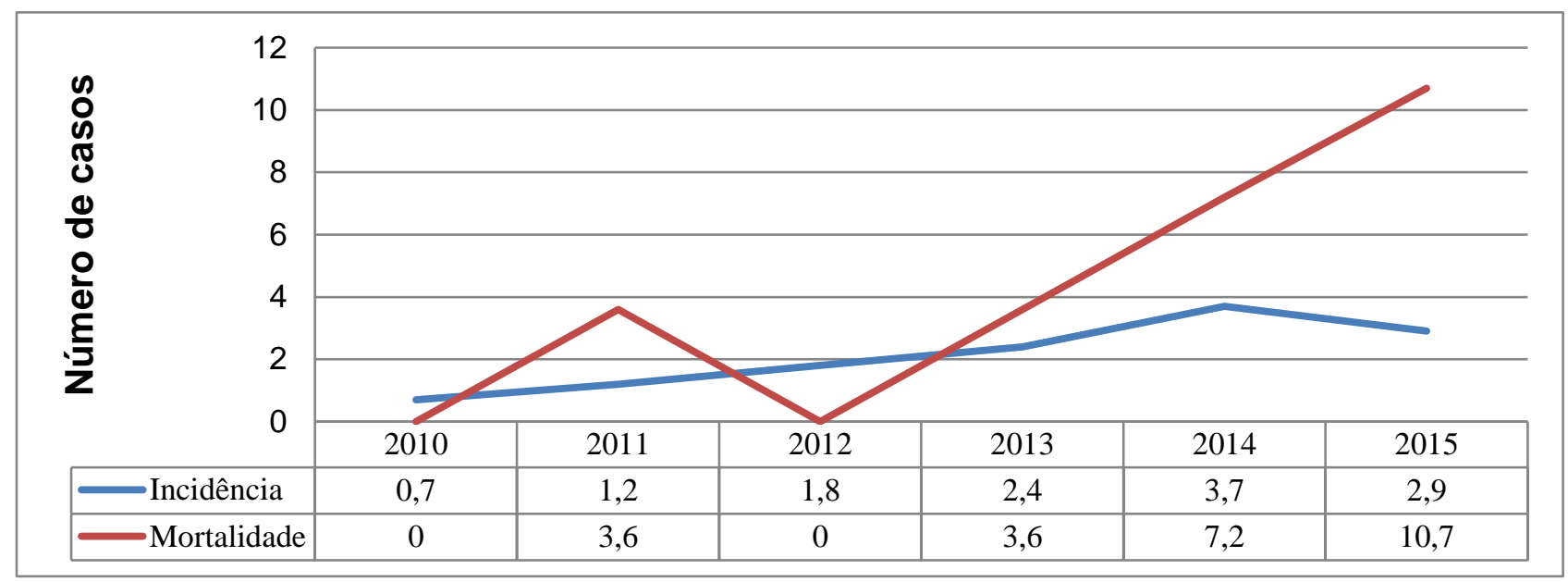

Fonte: SINAN/SINASC, 2016.

De acordo com a tabela 1, verifica-se que a maioria das gestantes com sífilis estavam entre 25 a 44 anos $(30,7 \%)$ exceto no biênio 2010-2011, prevalecendo mães adolescentes de 13 a 19 anos (38,5\%). Observa-se também que, independente do biênio, houve predomínio de mulheres pardas $(72,8 \%)$, com ensino fundamental $(54,2 \%)$, residentes em zona urbana $(89,6 \%)$, que realizaram o pré-natal $(82,3 \%)$, com diagnóstico de sífilis gestacional $(58,6 \%)$ por meio do VDRL reagente no parto/curetagem $(86,4 \%)$ e teste confirmatório $(49,6 \%)$. 
Tabela 1 - Distribuição das variáveis sociodemográficas, diagnóstico, tratamento da sífilis em gestantes e do parceiro, Rondônia, 2010 - 2015.

\begin{tabular}{|c|c|c|c|c|c|c|c|c|}
\hline \multirow{3}{*}{ Variável } & \multicolumn{8}{|c|}{ Biênio } \\
\hline & \multicolumn{2}{|c|}{$2010 / 2011$} & \multicolumn{2}{|c|}{$2012 / 2013$} & \multicolumn{2}{|c|}{$2014 / 2015$} & \multicolumn{2}{|c|}{$2010 / 2015$} \\
\hline & $\mathrm{n}=52$ & $\%$ & $\begin{array}{c}n=1 \\
11\end{array}$ & $\%$ & $n=182$ & $\%$ & $n=345$ & $\%$ \\
\hline \multicolumn{9}{|l|}{ Idade } \\
\hline 13-19 anos & 20 & 38,5 & 36 & 32,5 & 40 & 22 & 96 & 27,8 \\
\hline $20-24$ anos & 15 & 28,8 & 32 & 28,8 & 59 & 32,4 & 106 & 30,7 \\
\hline 25-44 anos & 17 & 32,7 & 43 & 38,7 & 83 & 45,6 & 143 & 41,5 \\
\hline \multicolumn{9}{|l|}{ Cor } \\
\hline Parda & 30 & 57,7 & 83 & 74,7 & 138 & 75,8 & 251 & 72,8 \\
\hline Branca & 6 & 11,5 & 13 & 11,7 & 15 & 8,2 & 34 & 9,8 \\
\hline Preta & 5 & 9,6 & 4 & 3,6 & 17 & 9,4 & 26 & 7,5 \\
\hline Ignorado & 11 & 21,2 & 11 & 10 & 12 & 6,6 & 34 & 9,9 \\
\hline \multicolumn{9}{|l|}{ Escolaridade* } \\
\hline $\begin{array}{l}\text { Ens. } \\
\text { Fundamental }\end{array}$ & 24 & 46 & 59 & 53,1 & 104 & 57,1 & 187 & 54,2 \\
\hline Ensino Médio & 11 & 21,2 & 29 & 26,1 & 49 & 27 & 89 & 25,7 \\
\hline Ensino Superior & 1 & 2 & 4 & 3,6 & 1 & 0,5 & 6 & 1,8 \\
\hline Ignorado & 16 & 30,8 & 19 & 17,2 & 28 & 15,4 & 63 & 18,3 \\
\hline \multicolumn{9}{|c|}{ Local de residência } \\
\hline Zona urbana & 47 & 90,3 & 100 & 90 & 162 & 89 & 309 & 89,6 \\
\hline Zona rural & 5 & 9,7 & 11 & 9,9 & 20 & 11 & 36 & 10,4 \\
\hline \multicolumn{9}{|l|}{ Realizou pré-natal } \\
\hline Sim & 43 & 82,6 & 92 & 82,8 & 149 & 81,8 & 284 & 82,5 \\
\hline Não & 6 & 11,6 & 11 & 10 & 28 & 15,4 & 45 & 12,7 \\
\hline Ignorado & 3 & 5,8 & 8 & 7,2 & 5 & 2,8 & 18 & 4,8 \\
\hline \multicolumn{9}{|c|}{ Diagnóstico Materno } \\
\hline Durante pré-natal & 22 & 42,3 & 72 & 64,8 & 108 & 59,3 & 202 & 58,6 \\
\hline $\begin{array}{l}\text { No momento do } \\
\text { parto/curetagem }\end{array}$ & 18 & 34,6 & 28 & 25,2 & 52 & 28,5 & 98 & 28,4 \\
\hline Após o parto & 11 & 21,1 & 8 & 7 & 17 & 9,5 & 36 & 10,4 \\
\hline Ignorado & 1 & 2 & 3 & 3 & 5 & 2,7 & 9 & 2,6 \\
\hline \multicolumn{9}{|c|}{ VDRL parto/curetagem } \\
\hline Reagente & 41 & 78,8 & 101 & 91 & 156 & 85,8 & 298 & 86,4 \\
\hline Não reagente & 1 & 2 & 4 & 3,6 & 3 & 1,6 & 8 & 2,3 \\
\hline Não realizado & 8 & 15,4 & 3 & 2,7 & 20 & 11 & 31 & 9 \\
\hline Ignorado & 2 & 3,8 & 3 & 2,7 & 3 & 1,6 & 8 & 2,3 \\
\hline \multicolumn{9}{|c|}{ Teste confirmatório } \\
\hline Reagente & 23 & 44,3 & 20 & 18 & 128 & 70,3 & 171 & 49,6 \\
\hline Não reagente & 1 & 2 & 2 & 1,6 & 8 & 4,4 & 11 & 2,6 \\
\hline Não realizado & 25 & 48 & 84 & 75,4 & 33 & 17,6 & 140 & 40,8 \\
\hline Ignorado & 3 & 5,7 & 6 & 5 & 14 & 7,7 & 23 & 7 \\
\hline \multicolumn{9}{|c|}{ Tratamento gestante } \\
\hline Adequado & 10 & 19,2 & 12 & 10,8 & 53 & 29,2 & 75 & 21,7 \\
\hline Inadequado & 18 & 34,6 & 63 & 56,7 & 91 & 50 & 172 & 50 \\
\hline Não realizado & 13 & 25 & 28 & 25,2 & 32 & 17,6 & 73 & 21,1 \\
\hline Ignorado & 11 & 21,2 & 8 & 7,3 & 6 & 3,2 & 25 & 7,2 \\
\hline \multicolumn{9}{|c|}{ Tratamento parceiro } \\
\hline Sim & 12 & 23 & 15 & 13,5 & 65 & 35,7 & 92 & 26,6 \\
\hline Não & 23 & 44,3 & 81 & 73 & 98 & 53,8 & 202 & 58,6 \\
\hline Ignorado & 17 & 32,7 & 15 & 13,5 & 19 & 10,5 & 51 & 14,8 \\
\hline
\end{tabular}

Fonte: SINAN, 2016. Nota: *Escolaridade: ensino fundamental de $1^{1}$ a $8^{\underline{a}}$ série; ensino médio de $1^{\circ}$ ao $3^{\circ}$ ano; ensino superior com graduação completa e incompleto.

Parte significativa dessas gestantes (48\% no biênio 2010-2011 e 75,7\% em 2012-2013) não realizou o teste confirmatório. Em relação ao tratamento, metade das gestantes com sífilis receberam tratamento inadequado $(50 \%)$ e os seus parceiros não foram tratados $(58,6 \%)$. Destacam-se os elevados percentuais de subregistros, principalmente no que concerne a escolaridade $(18,3 \%)$ e tratamento do parceiro $(14,8 \%)$.

A Tabela 2 mostra que a maiorias dos recém-nascidos tiveram o exame de VDRL reagente $(78,8 \%)$, não foram submetidas ao exame do líquor (76,2\%), mesmo quando em 2010-2011 não tenha ocorrido casos 
reagentes. Verifica-se também que a maior parte dessas crianças tiveram diagnóstico conclusivo de SC recente $(82,6 \%)$ evoluíram vivas $(89,3 \%)$ e assintomáticas $(76 \%)$. Ressalta-se o elevado numero de registros "ignorado", principalmente nas variáveis: exame de líquor (15\%), diagnóstico clínico $(7,2 \%)$ e evolução dos casos $(8,1 \%)$.

Tabela 2 - Distribuição dos resultados laboratoriais, diagnóstico e evolução dos casos de sífilis congênita, Rondônia, no período de 2010 - 2015.

\begin{tabular}{|c|c|c|c|c|c|c|c|c|}
\hline \multirow{3}{*}{ Variáveis } & \multicolumn{8}{|c|}{ Biênio } \\
\hline & \multicolumn{2}{|c|}{ 2010/2011 } & \multicolumn{2}{|c|}{ 2012/2013 } & \multicolumn{2}{|c|}{$2014 / 2015$} & \multicolumn{2}{|c|}{$2010 / 2015$} \\
\hline & $\begin{array}{l}n=5 \\
2\end{array}$ & $\%$ & $\mathrm{n}=111$ & $\%$ & $n=182$ & $\%$ & $\mathrm{n}=345$ & $\%$ \\
\hline \multicolumn{9}{|l|}{ VDRL criança } \\
\hline Reagente & 43 & 82,7 & 83 & 75 & 146 & 80,3 & 272 & 78,8 \\
\hline Não reagente & 3 & 5,7 & 19 & 17,2 & 25 & 13,7 & 47 & 13,6 \\
\hline Não realizado & 1 & 2 & 7 & 6,4 & 2 & 1 & 10 & 2,6 \\
\hline Ignorado & 5 & 9,6 & 2 & 1,4 & 9 & 5 & 16 & 5 \\
\hline \multicolumn{9}{|c|}{ Exame de Líquor } \\
\hline Reagente & - & - & 1 & 1 & 1 & 0,6 & 2 & 0,5 \\
\hline Não reagente & 11 & 21,2 & 7 & 6,3 & 11 & 6 & 29 & 8,3 \\
\hline Não realizado & 21 & 40,3 & 86 & 77,4 & 156 & 85,7 & 263 & 76,2 \\
\hline Ignorado & 20 & 38,5 & 17 & 15,3 & 14 & 7,7 & 51 & 15 \\
\hline \multicolumn{9}{|c|}{ Diagnóstico Clínico } \\
\hline Assintomático & 12 & 23 & 87 & 78,3 & 163 & 89,6 & 262 & 76 \\
\hline Sintomático & 5 & 9,6 & 4 & 3,6 & 10 & 5,4 & 19 & 5,5 \\
\hline Não se aplica & 33 & 63,5 & 4 & 3,6 & 2 & 1 & 39 & 11,3 \\
\hline Ignorado & 2 & 3,7 & 16 & 14,5 & 7 & 4 & 25 & 7,2 \\
\hline \multicolumn{9}{|c|}{ Diagnóstico final da criança } \\
\hline SC recente & 51 & 98 & 101 & 91 & 133 & 73 & 285 & 82,6 \\
\hline Descartados & 1 & 2 & 10 & 9 & 49 & 27 & 60 & 17,4 \\
\hline \multicolumn{9}{|c|}{ Evolução dos casos } \\
\hline \multirow{4}{*}{$\begin{array}{l}\text { Vivos } \\
\text { Óbitos por SC } \\
\text { Óbitos por } \\
\text { outras causas }\end{array}$} & 41 & 78,7 & 101 & 90 & 166 & 91,3 & 308 & 89,3 \\
\hline & 1 & 2 & 1 & 1 & 5 & 2,7 & 7 & 2 \\
\hline & 1 & 2 & 1 & 1 & 1 & 0,5 & 3 & 0,6 \\
\hline & 9 & 17,3 & 8 & 8 & 10 & 5,5 & 27 & 8,1 \\
\hline
\end{tabular}

Fonte: SINAN, 2016

\section{DISCUSSÃO}

A incidência e mortalidade por Sífilis Congênita (SC) em Rondônia teve um aumento significativo entre os anos de 2010 e 2015. Esse fenômeno também foi documentado na capital do estado no período de 2009 a 2014 onde houve um acréscimo de 0,92 casos a 8,65/1.000 nascidos vivos (MOREIRA KFA, et al., 2017). O aumento dessas taxas, sobretudo da mortalidade por SC, pode estar relacionado à melhoria das notificações desse agravo, em razão da qualificação dos profissionais de saúde da Atenção Primaria em Saúde dos 52 municípios a partir de 2014, quando se investiu no manejo das IST, e em especial a sífilis congênita (RONDONIA, 2015). Por outro lado, a elevação dessas taxas apontam que ainda é crucial o fortalecimento das ações de prevenção, detecção e tratamento precoce da sífilis materna por meio da articulação entre gestores, profissionais e comunidade, sobretudo no que diz respeito à melhoria nos indicadores de pré-natal e parto conforme preconizado (BRASIL, 2018; LAFETÁ KRG, 2016).

A taxa de incidência de SC em 2015 (2,9/1000 nascidos vivos) foi quatro vezes maior que 2010 (0,7/1000 nascidos vivos). Embora esta incidência no ano de 2015 seja inferior à taxa da região Norte $(4,4)$ e nacional $(6,5)$ ela está cinco vezes superior à meta de eliminação da SC, pactuada por 52 países da região das Américas, que é de 0,5/1000 nascidos vivos até 2030 (WHO, 2017; BRASIL, 2018). Por sua vez, a taxa de 
mortalidade em 2015 em Rondônia (10,7/100.000 nascidos vivos) foi superior à da região Norte (9,3/100.000 nascidos vivos) e do país (6,5/100.000 nascidos vivos) para o mesmo período.

Dos casos analisados, encontrou-se maior predomínio de sífilis entre mães com idade acima de 20 anos, pardas, com ensino fundamental e residentes em área urbana. Pesquisas realizadas no estado do Ceará, apontam uma maior vulnerabilidade de mulheres adultas, incluindo gestantes com sífilis que permaneceram com práticas sexuais desprotegidas, mesmo cientes das relações extraconjugais dos parceiros (COSTA CC, et al., 2013). É um contexto desafiado aos profissionais de saúde no país, sobretudo das equipes da Estratégia Saúde da Família, uma vez que grande parte dessas mulheres estão na fase reprodutiva, implicando em maior número de gestações em virtude da relação sexual desprotegida (SOUSA WN e BENITO LAO, 2019).

A significativa baixa escolaridade dos casos analisados pode estar relacionada ao baixo acesso à informação ou compreensão das gestantes sobre as medidas de prevenção da doença na gravidez (COSTA CC, et al., 2013). Um aspecto que chama a atenção é que, considerando-se o local de residência das mães na zona urbana, era esperado que a proximidade dos serviços de saúde facilitasse o diagnóstico precoce e acompanhamento destas gestantes. Entretanto, este fato sugere que a sífilis gestacional em Rondônia, não esteja restrito apenas à área de residência como encontrado por Souza AS e Sampaio LJ (2018) e Maeda ATN et al. (2018) e sim às iniquidades sócio geográficas da região amazônica (LEAL MC, et al., 2015).

Apesar dos recursos diagnósticos, o controle da doença ainda é um desafio de profissionais e gestores, sobretudo pela dificuldade de abordagem das IST na gestação e em alguns casos, pelo desconhecimento da gravidade dos efeitos em longo prazo na mãe e no Recém-Nascido (RN) (LAFETÁ KRG, 2016). Além disso, sabe-se que o diagnóstico tardio aumenta 1,68 vezes o risco de SC (MAGALHÃES DMS, 2013). Estudo sobre SC na capital de Porto Velho realizado entre 2009 a 2014, cerca de 60,10\% das gestantes foram diagnosticadas no pré-natal (MOREIRA KFA, et al., 2017). No presente estudo, $38 \%$ das mulheres foram diagnosticadas no momento do parto, na curetagem e/ou no pós-parto. Tal fato remete à importância do diagnóstico precoce e acompanhamento dos casos bem como a qualidade da assistência no pré-natal, às práticas do cuidado em saúde e a efetividade das capacitações realizadas para atualização dos profissionais (REZENDE E e BARBOSA NB, 2015).

Neste estudo, metade das gestantes $(50 \%)$ realizou o tratamento da sífilis inadequadamente e uma parcela nem sequer realizou (21,1\%). Este resultado se assemelha ao encontrado por Moreira KFA et al. (2017) na capital do estado de Rondônia, onde $55,1 \%$ das 385 gestantes diagnosticadas no pré-natal, foram tratadas incorretamente. Este resultado reforça a precária abordagem às gestantes no pré-natal, considerando que nos 52 municípios de Rondônia, 69,5\% das mulheres grávidas em 2010 foram submetidas ao exame do VDRL e destas, $51,0 \%$ receberam o resultado em até 15 dias (RONDONIA, 2015). Estes achados se assemelham a um estudo no estado de Goiás nos anos de 2009 a 2012, onde 43,3\% das gestantes notificadas receberam tratamento inadequado e 32,5\% não realizou tratamento (REZENDE E e BARBOSA NB, 2015). Tais dados revelam iniquidades da atenção ao pré-natal em relação à vigilância e controle desse agravo em diferentes regiões do país.

O tratamento inadequado da sífilis ou a não realização deste, aumenta os casos de reinfecção nas gestantes, diminui as chances de interrupção da cadeia de transmissão e ainda contribui para o aumento dos casos de Transmissão Vertical da doença (WANG YAJIE, et al., 2019; WHO, 2017). Portanto, se faz necessária a reafirmação da importância do tratamento precoce e oportuno, bem como a busca ativa dos parceiros sexuais, seja pela APS ou pela vigilância epidemiológica (REZENDE E e BARBOSA NB, 2015).

Nesse estudo, uma significativa parcela das genitoras não realizou o teste laboratorial confirmatório no momento do parto, embora este exame seja preconizado pelos protocolos clínicos em nível mundial (WHO, 2017). Também houve falha das recomendações protocolares para diagnóstico da SC em tempo oportuno e adequado já que a distribuição dos resultados laboratoriais das crianças foi demorada e prejudicou a tomada de decisão para iniciar tratamento. Em Rondônia, isso pode ser reflexo de possíveis dificuldades estruturais ou de acesso para realização dos exames, conforme recomendado mundialmente (WHO, 2017). 
Nas crianças expostas ao treponema pallidum o exame mais realizado foi o VDRL enquanto a punção liquórica, também essencial para o diagnóstico, não foi realizada na maior parte dos casos. Sabe-se que somente a titulagem do VDRL não indica uma infecção congênita, o que evidencia uma baixa qualidade do diagnóstico da SC (GUERRERO M, LUCIANI K, 2019). Resultados semelhantes foram descritos por Magalhães DMS et al. (2013) no Distrito Federal de 2009 a 2010 e por Rezende E e Barbosa NB (2015) no estado de Goiás entre os anos de 2009 a 2012. Ambos estudos, identificaram que quase metade dos RN não realizaram qualquer teste e/ ou qualquer exame laboratorial complementar

A maioria das crianças com desfecho de SC teve diagnóstico classificado como recente e clinicamente assintomáticas como relatado na literatura (GUERRERO M e LUCIANI K, 2019). No estudo de Sousa AS e Sampaio LJ (2019) feito na capital Porto Velho, 98,7\% das crianças tiveram diagnóstico em até seis dias de vida, ou seja diagnóstico recente, entretanto deste percentual, houve quatro óbitos. Esse dado revela que o fato de o diagnóstico ter ocorrido em tempo oportuno não correspondeu ao efetivo acompanhamento da maioria dos casos. É de praxe que todos os critérios (epidemiológicos, clínicos e laboratoriais) sejam cumpridos em conjunto e que todas as crianças com SC sejam acompanhadas com atendimento laboratorial, neurológico e fonoaudiólogo por dois anos (BRASIL, 2019).

Neste estudo, não foi avaliado o seguimento de $89,3 \%$ dos casos de crianças com SC. Este achado encontra semelhança ao estudo de Lafetá KRG et al. (2016), com crianças atendidas em um serviço de referência pediátrico, onde $79,6 \%$ delas não foram sequer referenciadas. Outro estudo realizado na cidade de Montes Claros-MG identificou que dos 54 recém-nascidos considerados com sífilis congênita, apenas $51,8 \%$ foram tratados conforme protocolo do Ministério da Saúde e de 16,1\% evoluíram para natimorto ou abortamento (NONATO MS et al., 2015).

Chama atenção o significativo número de subnotificações e subregistros (ignorados) da SC. Tal fato pode ser atribuído a não realização da busca ativa dos casos bem como o inadequado manuseio e registro das fichas de notificação. Esta problemática foi levantada em todo país no perfil epidemiológico da SC entre 2008 a 2014. Variáveis como a escolaridade materna $(29,6 \%)$ e tratamento do parceiro $(22,4 \%)$ tiveram os maiores percentuais de casos ignorados/brancos (SOUZA WN, BENITO LAO, 2016). Verifica-se que informações essenciais dos casos em investigação são perdidas e tornando as ações de controle insipientes diante da velocidade e crescimento dos casos (LAFETÁ KRG et. al., 2016).

A limitação desta análise recaiu sobre a incompletude das fichas de notificações que por sua vez, impactam na qualidade dos registros e na contestação da validade dos mesmos. Por outro lado, o SINAN é a base oficial de informações de agravos a serviço do planejamento das gestões em todo território nacional. Assim, os resultados poderão nortear a qualificação das notificações concomitante à capacitação dos profissionais que atuam na atenção pré-natal, contribuindo para eliminação/redução da sífilis materna e SC no estado. Contudo, este problema só será resolvido na medida em que a vigilância dos dados gerados em cada município for efetiva e oportuna. Para tanto, é urgente que se adotem medidas efetivas no processo de notificações da SC para reduzir o elevado número de informações inconsistentes tidas como "ignorado" por meio de estratégias como busca ativa dos casos e adequado manuseio/registro das fichas de notificações, apoiadas na criação de comitês de investigação de sífilis congênita em cada município, para monitorar a doença em tempo oportuno.

\section{CONCLUSÃO}

Nesse estudo, houve elevadas taxas de incidência e mortalidade por SC em Rondônia no período de 2010 a 2015, um reflexo das fragilidades da assistência pré-natal, pelo tratamento inadequado da sífilis materna e diagnóstico tardio da SC no parto e puerpério. É preciso que gestores estaduais e municipais invistam em políticas de educação permanente e continuada para fortalecer a corresponsabilização dos profissionais envolvidos na linha de cuidado à saúde materno-infantil, por se tratar de um agravo evitável, curável, de baixo custo e crucial para a redução e controle dos casos de SC na região. 


\section{AGRADECIMENTOS}

Ao professor Dro Edson Farias e Professora Me. Ellen Petean da Universidade Federal de Rondônia e ao professor Natanael Arruda, coordenador do Núcleo de Infecções Sexualmente Transmissíveis/Aids/Vírus da Imunodeficiência Humana da Agência de Vigilância em Saúde /Secretaria de Saúde do Estado de Rondônia.

\section{REFERÊNCIAS}

1.BOTTURA BR et al. Perfil epidemiológico da sífilis gestacional e congênita no Brasil - período de 2007 a 2016. Arquivo Médico Hospitalar da Faculdade de Ciência Médicas da Santa Casa, São Paulo, 64 (2): 69-75.

2.BRASIL. Ministério da Saúde. Secretaria de vigilância em saúde. Programa Nacional de DST, Aids e Hepatites Virais. Boletim Epidemiológico - Sífilis, 2018.

3.BRASIL. Ministério da Saúde. Secretaria de Vigilância em Saúde. Departamento de Doenças de Condições Crônicas e Infecções Sexualmente Transmissíveis. Protocolo Clínico e Diretrizes Terapêuticas para Prevenção da Transmissão Vertical do HIV, Sífilis e Hepatites Virais, 2019.

4. SOUSA AS, SAMPAIO LJ. Perfil epidemiológico dos casos de sífilis congênita notificados no município de Porto Velho/Rondônia no período de 2013 a 2017. Jornal Brasileiro de Revisão de Saúde, 2019; 2(3): 1609-1615.

5.COSTA CC et al. Sífilis congênita no Ceará: análise epidemiológica de uma década. Revista Escola de Enfermagem, 2013; 47 (1): 152-9.

6.GUERRERO, M, LUCIANI, K. Sífilis congénita: características y calidad de atención en el Complejo Hospitalario Metropolitano Dr. Arnulfo Arias Madrid Dr. Arnulfo Arias Madrid. Pediátr. Panamá, 2019; 48 (1): 5-11.

7.LAFETÁ KRG et al. Sífilis materna e congênita, subnotificação e difícil controle. Revista Brasileira de Epidemiologia, 2016; 19 (1): 63-74.

8.LEAL MC et al. Atenção ao pré-natal e parto em mulheres usuárias do sistema público de saúde residentes na Amazônia Legal e no Nordeste, Brasil 2010, Revista Brasileira de Saúde Materno Infantil, $2015 ; 15$ (1): 91 -104.

9. MOREIRA KFA et al. Perfil dos casos notificados de sífilis congênita. Cogitare Enfermagem, 2017; 22(2) e48949.

10. MAGALHÃES DMS et al. Sífilis materna e congênita: ainda um desafio. Caderno de Saúde Pública, $2013 ; 29$ (6): 1109-1120.

11. MAEDA ATN et al. Perfil clínico e epidemiológico das gestantes com sífilis e sífilis congênita no município de Cacoal, Rondônia, Brasil, 2017 a 2016. Revista Eletrônica FACIMEDIT, 2018; 7(1): 41-50.

12.NONATO MS et al. Sífilis na gestação e fatores associados à sífilis congênita em Belo Horizonte- MG, $2010-2013$.

Epidemiologia e Serviços de Saúde, 2015; 24 (4): 681-694.

13.RONDÔNIA. Governo do Estado de Rondônia. Agência Estadual de Vigilância Epidemiológica. Secretaria de Estado da Saúde. Relatório Anual de Gestão, Porto Velho, Rondônia, 2015; 358p.

14.REZENDE EMA, BARBOSA NB. A sífilis congênita como indicador da assistência de pré-natal no estado de Goiás.

Revista de Atenção Primária Saúde, 2015; 18 (2): 220-232.

15.SILVA, LMC et al. Sífilis congênita no estado do Pará-Brasil, 2007 a 2016. Revista Eletrônica Acervo Saúde, 2019; 24 (1003): 1-10.

16.SHAHROOK S et al. Strategies of testing for syphilis during pregnancy. Cochrane Database of Systematic Reviews. John Wiley \& Sons, 2014; (1): 37-43.

17.SOUSA OC et al. Sífilis congênita: o reflexo da assistência pré-natal na Bahia. Jornal de Brasileiro de Revisão em Saúde, 2019; 2(6) 1356-1376.

18.SOUZA WN, BENITO LAO. Perfil epidemiológico da sífilis congênita no Brasil no período de 2008 a 2014. Universitas: Ciências da Saúde, 2016; 14 (2): 1-8.

19.WANG, YAJIE et al. Congenital Syphilis Transmitted from Mother to Infant - Suzhou, China, 2011-2014.2019; 68(10): 247-250.

20. WHO (World Health Organization). Elimination of mother-to-child transmission of HIV and syphilis in the Americas. Update 2016. Washington, D.C. PAHO; 2017. 